\title{
MICROTEXTURAL CHARACTERIZATION OF A BERYLLIUM WELDMENT
}

\author{
STUART I. WRIGHT and JAMES D. COTTON \\ Los Alamos National Laboratory, Los Alamos, NM 87545 (USA)
}

(Received 14 June 1994)

\begin{abstract}
Local grain orientations in a beryllium weldment have been characterized using an automatic technique based on computer indexing of electron backscatter diffraction patterns. The results indicate that many weld grains are epitaxially nucleated from the base metal. The number of grains allowed further growth is quickly reduced based on their orientations relative to the macroscopic growth direction. Grains with $<1010\rangle$ crystallographic directions parallel to the macroscopic growth direction tend to dominate. Examination of the spatial distribution of orientation within individual grains shows that, while the macroscopic growth direction changes during solidification, the crystallographic orientation does not. That is, substantial deformation of the weld grains does not occur during grain growth. Analyses adjacent to the centerline fracture indicate that the fracture plane correlates best with $\{11 \overline{2} 0\}$ crystallographic planes.
\end{abstract}

KEY WORDS: Microtexture, beryllium, electron beam welding.

\section{INTRODUCTION}

Beryllium, with a density of $1.85 \mathrm{~g} / \mathrm{cc}$, is the lightest structural metal. This property makes its specific properties remarkably attractive. For example, its density-compensated modulus its approximately six times that of $\mathrm{Al}, \mathrm{Ti}$ or steel, making it ideal for rigid flight structures. Furthermore, it has a melting point of nearly $1300^{\circ} \mathrm{C}$, which is also much greater than the other light metals, and thus more suitable for elevated temperature applications.

In spite of such desirable properties, Be has found only limited use in structural applications, partly due to its poor autogenous weldability. This is usually manifested as longitudinal cracking along the welded joints and is severe enough that alternative joining methods have been sought (Grant, 1979; Olson and Liby, 1979; Switz, 1979). However, none of the alternative methods (brazing, diffusion bonding, mechanical fasteners) can provide all the advantages inherent in the fusion welding process. Thus, there is a large impetus to understand the sources of weld cracking in Be and what can be done to ameliorate them.

One possible source of longitudinal, or centerline, weld cracking in $\mathrm{Be}$ is that resolidification of the weld produces a growth texture containing a larger than average proportion of low cleavage strength crystallographic planes at the centerline (Hauser et al., 1967). The preferred cleavage plane in $\mathrm{Be}$ is (0001) (Aldinger, 1979; Darwin and Buddery, 1960). Therefore, the objective of this work is to characterize the local crystallographic texture within a typical Be weldment and to identify any relationships 
Table 1 Impurity content (wppm) in ingot source beryllium used in this study.

\begin{tabular}{cccccccccc}
\hline$T i$ & $A l$ & $S i$ & $F e$ & $N i$ & $M n$ & $C u$ & $C r$ & $M g$ & $B e O$ \\
\hline 1500 & 1000 & 1700 & 1500 & 200 & 100 & 120 & 150 & 20 & 200 \\
\hline
\end{tabular}

with the fracture plane orientation and general microstructural features. In addition, the propensity for weld grain deformation (bending) during solidification was investigated.

\section{EXPERIMENTAL DETAILS}

\section{Starting Material and Welding Procedure}

Rolled, ingot-source beryllium sheet was obtained in the form of $2.5 \mathrm{~cm} \times 7.5 \mathrm{~cm}$ $\times 0.64 \mathrm{~cm}$ coupons for weld trials. The analyzed impurity content was typical of ingot source material and is shown in Table 1. An electron beam welding procedure was developed to produce full penetration bead-on-plate welds in a Hamilton Standard electron beam machine at $100 \mathrm{kV}, 15 \mathrm{~mA}$ and $25 \mathrm{~cm} / \mathrm{s}$ travel speed under a vacuum of $4 \times 10^{-5} \mathrm{~atm}$. A typical pass was then produced on an unconstrained coupon to minimize thermal stresses. Upon removal of the welded coupon a fracture was visually observed to run nearly the full length of the weld.

\section{Optical Metallography}

Representative transverse and short transverse sections were removed from the coupon center, metallographically prepared, and photographed in polarized light in the aspolished condition. These are shown in Figure 1.

\section{Microtexture Measurements}

Approximately 45,000 individual lattice orientation measurements were made on the short-transverse section. Spatially specific orientation measurements were made using a technique based on computer indexing of electron backscatter diffraction patterns (Adams et al., 1993; Wright, 1993). The measurements were obtained on a regular hexagonal grid with $25 \mu \mathrm{m}$ spacing between measurement points. At each point on the grid the orientation (in Euler angles after the manner of Bunge (1982)), the location ( $x, y$ coordinates) and a parameter quantifying the quality of the associated diffraction pattern were recorded.

A grain boundary map can be reconstructed from the measurements by considering the misorientation $(\omega)$ between neighboring points on the measurement grid. If the misorientation between two neighboring measurement points exceeds $5^{\circ}$ a thin line is drawn separating the two points. If the misorientation exceeds $15^{\circ}$ a thicker line is drawn. This results in the grain boundary map shown in Figure 2. Clearly, this reconstructed boundary map looks very similar to the micrograph shown in Figure 1b. This confirms that the orientational aspects of the microstructure in the measured plane have been correctly captured by the point-by-point orientation measurement technique.

In order to aid in the analysis of the data, the measurement region was partitioned into four distinct zones. Zone 1 contains all orientation measurements in the equiaxed grain structure region (starting microstructure). Zone 2 contains all measurements lying 

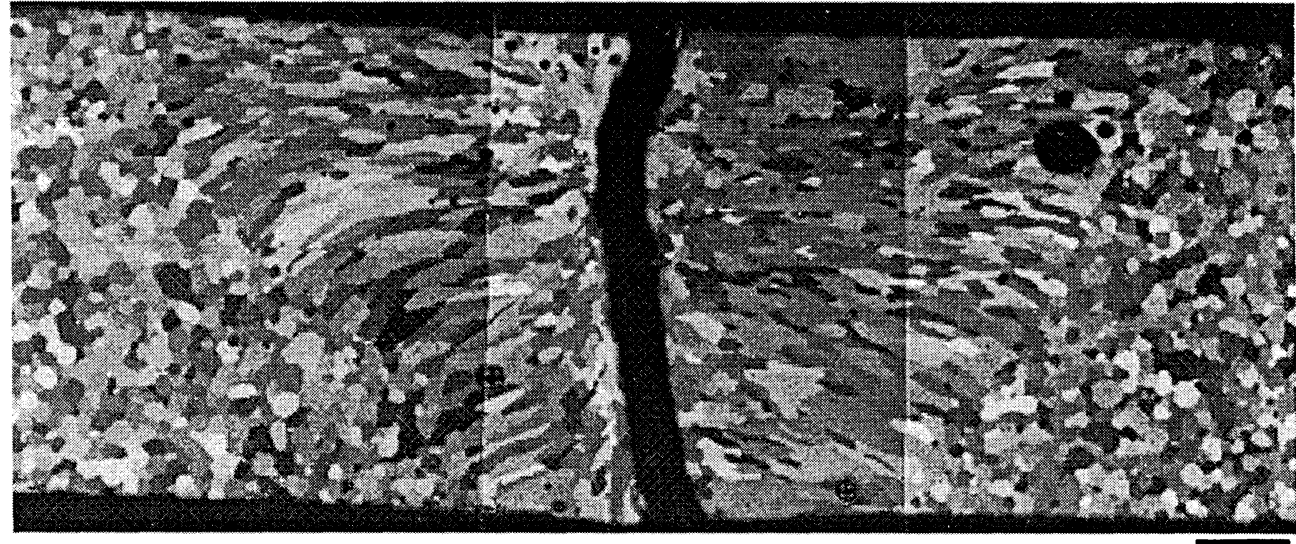

(a)

$1 \mathrm{~mm}$
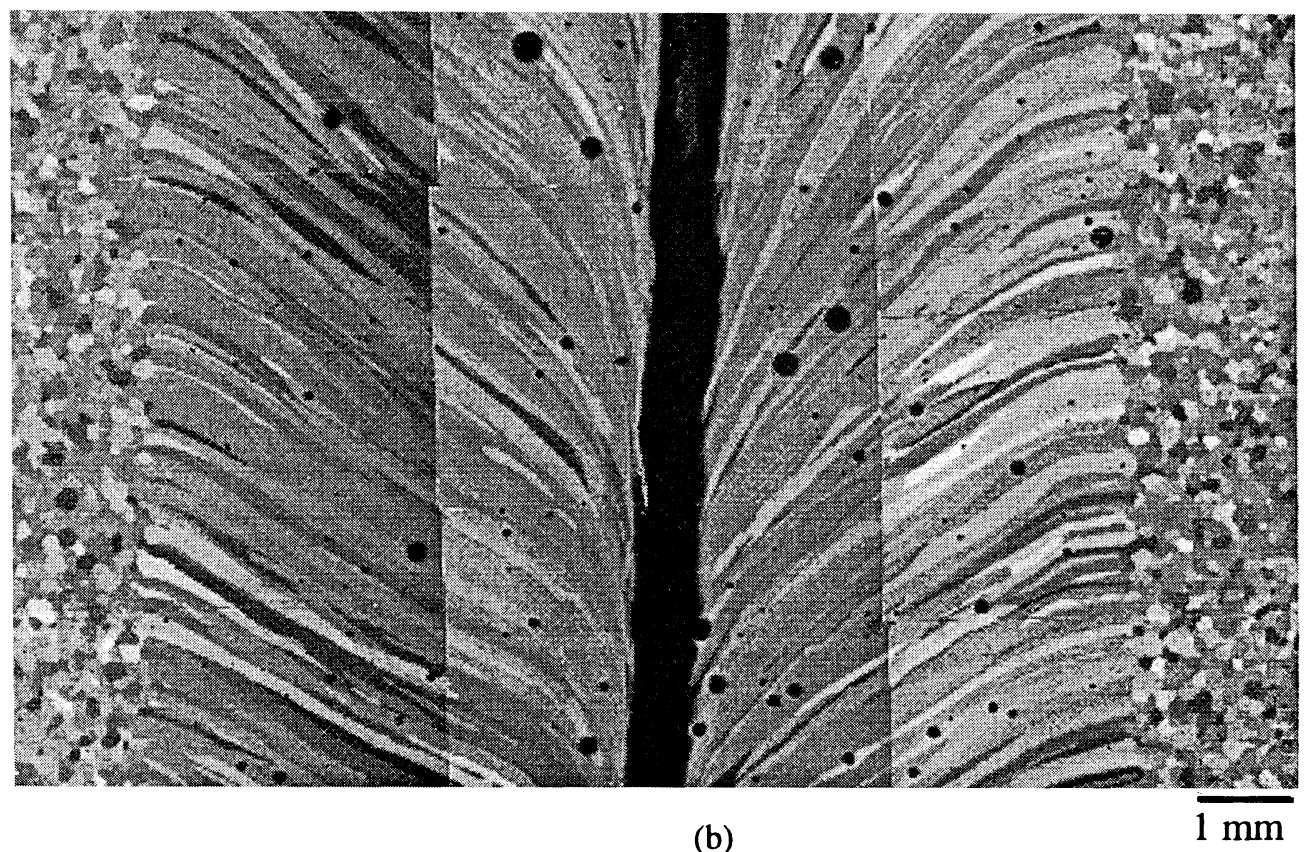

(b)

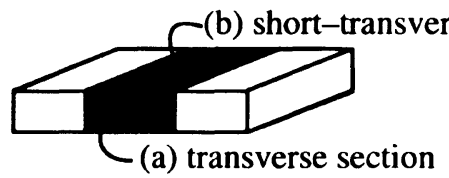

(c)

Figure 1 Optical micrographs of ingot source be electron beam weldment: (a) transverse section; (b) short-transverse section; (c) schematic of micrograph sections. 


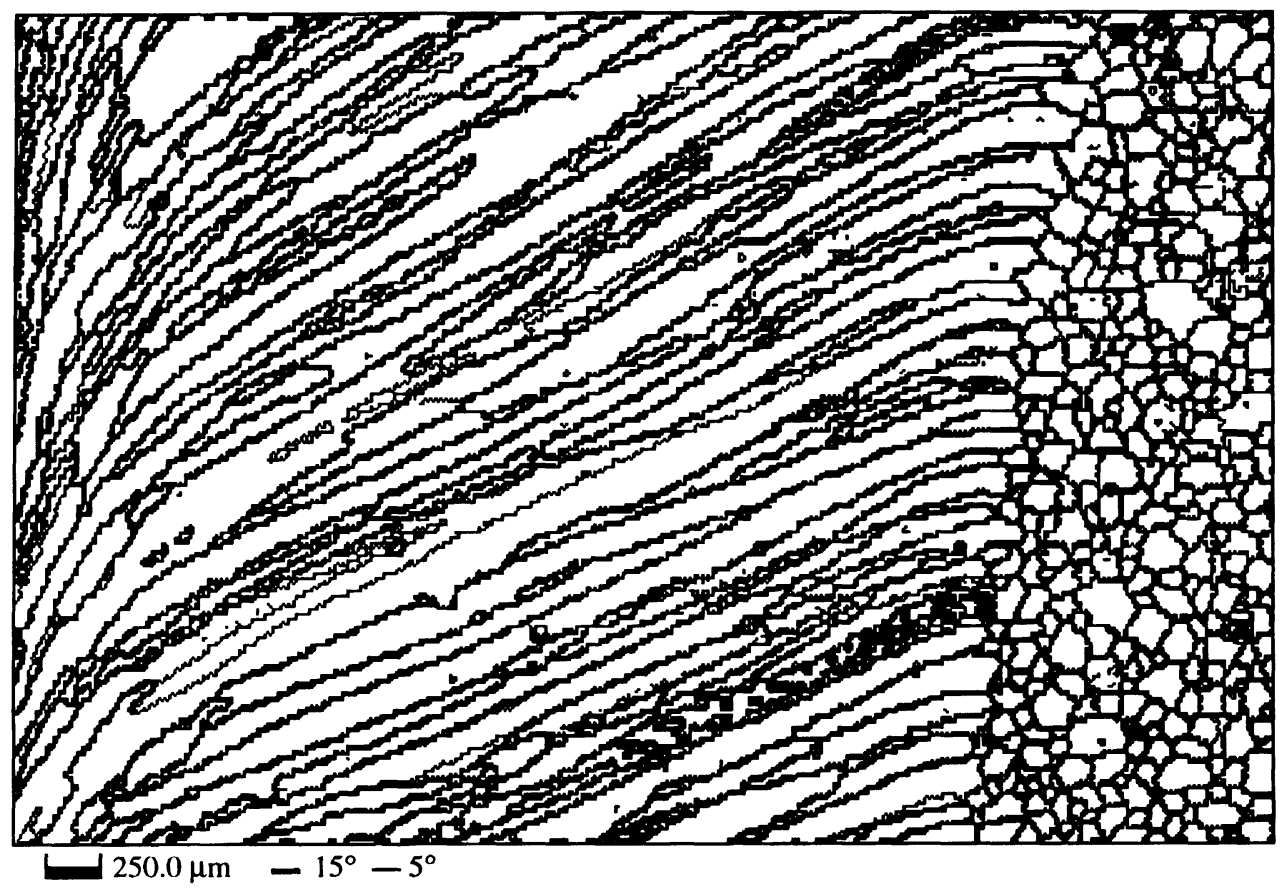

Figure 2 Grain boundary map reconstructed from orientation measurements.

just to the left of zone 1 and just before the elongated grains start their downward turn (i.e. $220 \mu \mathrm{m}$ left of the boundary separating the equiaxed grains from the elongated grains). Zone 4 contains all of the measurements in a region extending $510 \mu \mathrm{m}$ from the left edge of the measurement region. Zone 3 contains all measurements between zones 2 and 4. These zones are delineated in Figure 3. The results presented in the following section follow the partitioning scheme outlined here. In addition, two of the elongated grains (hereafter denoted grains A and B) were selected for individual microtexture analysis and are highlighted in Figure 3.

\section{RESULTS}

\section{Misorientation within Individual Grains}

The grains are delineated in Figure 2 through the reconstruction of grain boundaries by comparing the orientations of neighboring points on the measurement grid. If the misorientation between two neighboring points exceeds $5^{\circ}$ then a boundary segment is drawn. However, this only marks sharp changes in orientation. Diffuse changes in orientation (with respect to the scale of the spacing of the measurement array) will not be detected through the comparison of orientation between neighboring points. Thus, the grains appearing in the grain boundary map reconstruction could potentially have quite large spreads in orientation over their length. To better represent the overall spread of orientations within the selected A and B grains, all of the orientation measurements 


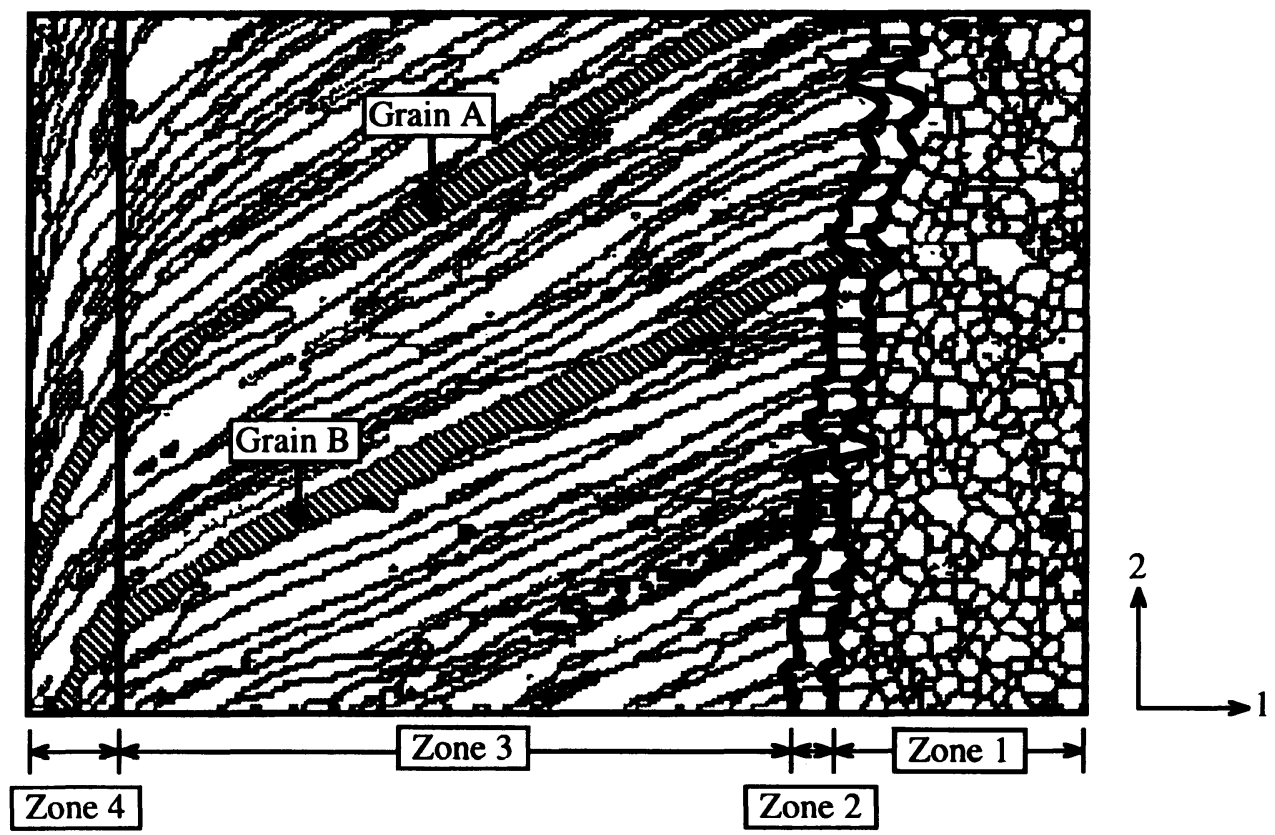

Figure 3 Schematic of the partitioning of the measurement region into four zones and selected grains.

were plotted as discrete pole figures, as shown in Figure 4. It should be noted that the two grains have similar orientations with respect to the rolling plane.

These pole figures show that the collective misorientations within grains A and B do not vary significantly within each grain. The scatter in orientation within each grain is shown in Figure 5. This figure was generated by comparing the misorientation between all points encompassed by a particular grain. In grain A $91 \%$ of the points lie within $2^{\circ}$ of each other and in grain B $95 \%$ of the points lie within $2^{\circ}$ of each other.

The spread in orientation does not give an indication of any systematic accumulation of misorientations along the grain length (however, it does provide a limit on the accumulation), such as would be observed in the case of grain "bending". To investigate this possibility, cumulative misorientation traces were constructed from the data of both grains $\mathrm{A}$ and $\mathrm{B}$. This was done by selecting a vertically centered starting point within each grain near the zone 2/zone 3 boundary. From these starting points, connecting lines were extended leftward within the grain to the end of the measurement region. These lines were also centered vertically within the grain. Measurements lying within $20 \mu \mathrm{m}$ of these lines were collected. The misorientation and distance of each measurement point, relative to the starting point, was calculated. The results for grain $\mathrm{A}$ are shown in Figure 6 (grain B showed very similar results). Although there are some slight trends in spatially correlated misorientation along the trace lines, no noteworthy cumulative change in grain orientation occurs along the length of the grain.

\section{Orientation Distributions in the Weld Zones}

As described above, the analysis region was divided into four zones. Discrete pole figures were constructed for each zone from the orientation measurements for the principal 
Grain A

1219 orientations

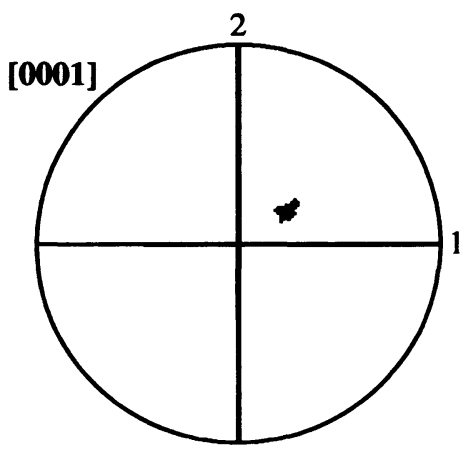

Grain B

1712 orientations

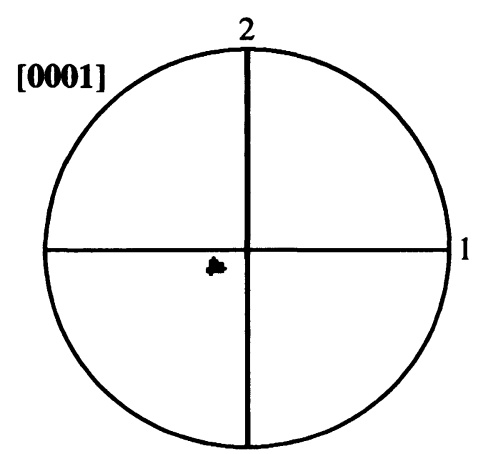

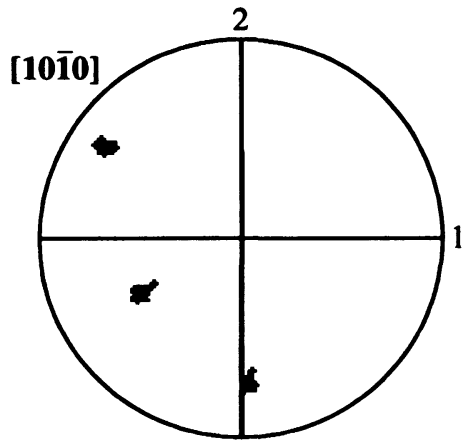

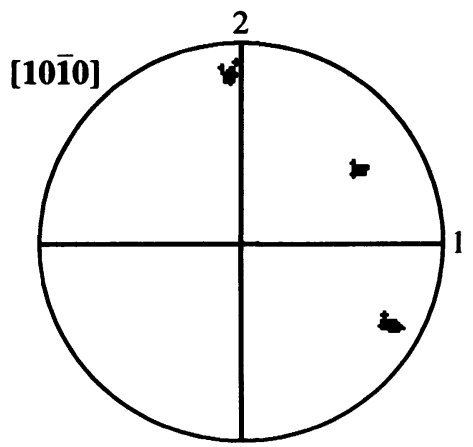

Figure 4 Discrete pole figures for all orientation measurements contained in grains A and B.

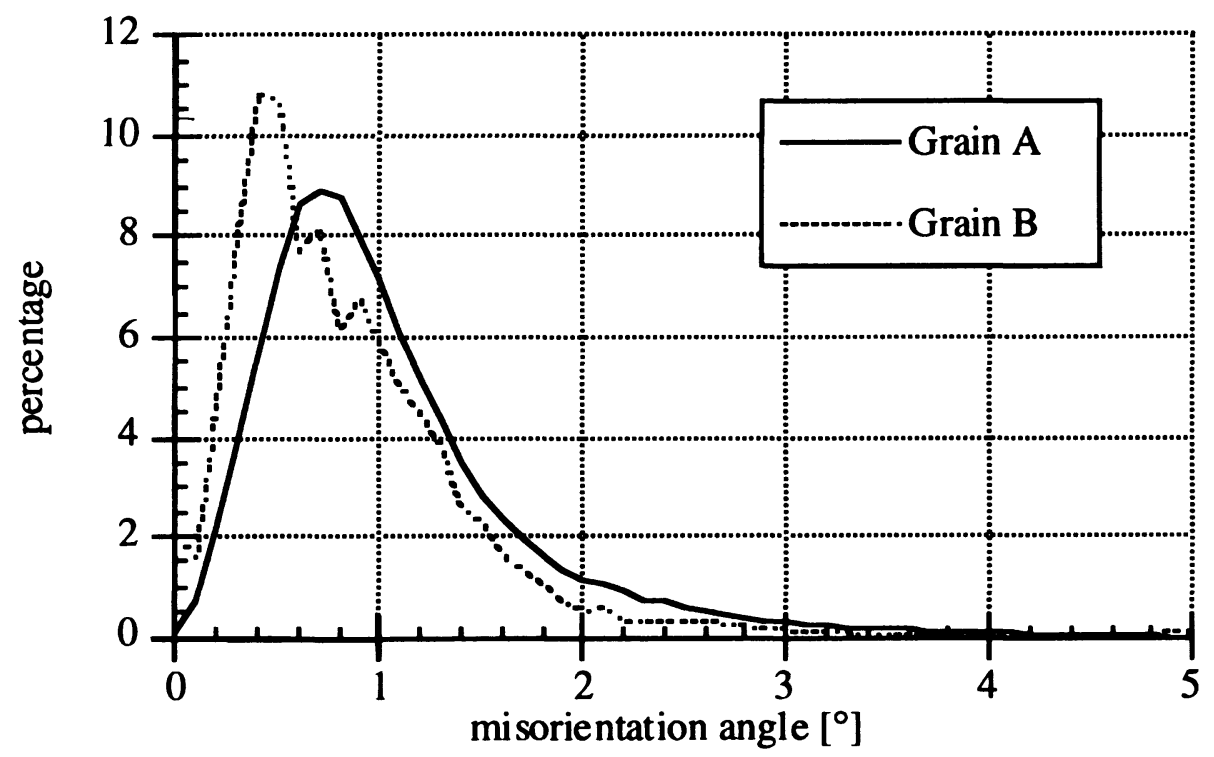

Figure 5 Spread of local misorientation in grains A and B. 


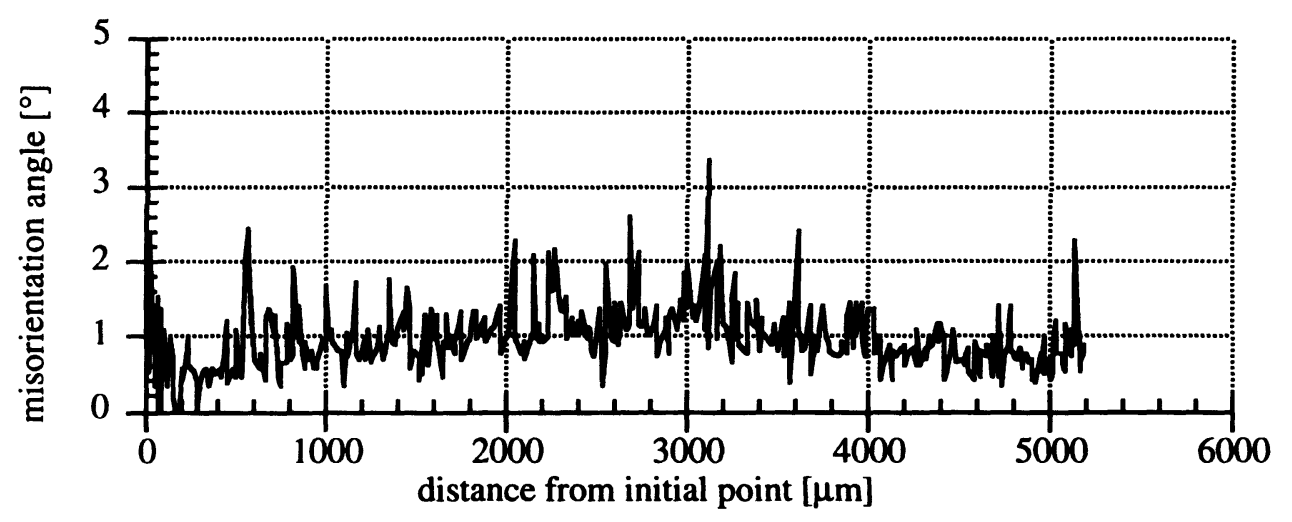

Figure 6 Misorientation trace in the interior of grain A.

<hkil> directions of beryllium. The (0001) discrete pole figures are shown in Figure 7 along with the number of measurements in each zone.

Orientation distribution functions (ODFs) for each of the four zones were calculated using harmonic analysis after the manner of Bunge (1982). Each orientation was represented as a Gaussian with a $5^{\circ}$ half-width (Wagner et al., 1981). All calculations were carried out to an order of $l=12$. Hexagonal crystal symmetry and triclinic sample symmetry were enforced. The ODF calculations are summarized in Table 2.

This data shows that the textures become progressively sharper as one moves towards the weld centerline. The band of intensity which is initially present in zone 1 (the initial rolling texture) is observed to rotate at the transition from zone 2 to zone 3 , essentially following the apparent grain growth direction. It should be noted that while there are significant numbers of orientation measurements in each zone the number of actual grains in each zone is quite small. Inspection of the grain map (Figure 2) reveals that there are approximately 367 grains in zone 1, 41 grains in zone 2, 127 grains in zone 3 and 54 grains in zone 4.

Inverse pole figures were calculated for each zone using the harmonic analysis described previously. Inverse pole figures give the distribution of crystal directions aligned with a particular sample direction. In this case, the sample direction selected for each zone was one aligned with the long axes of the grains in the zones (i.e. the
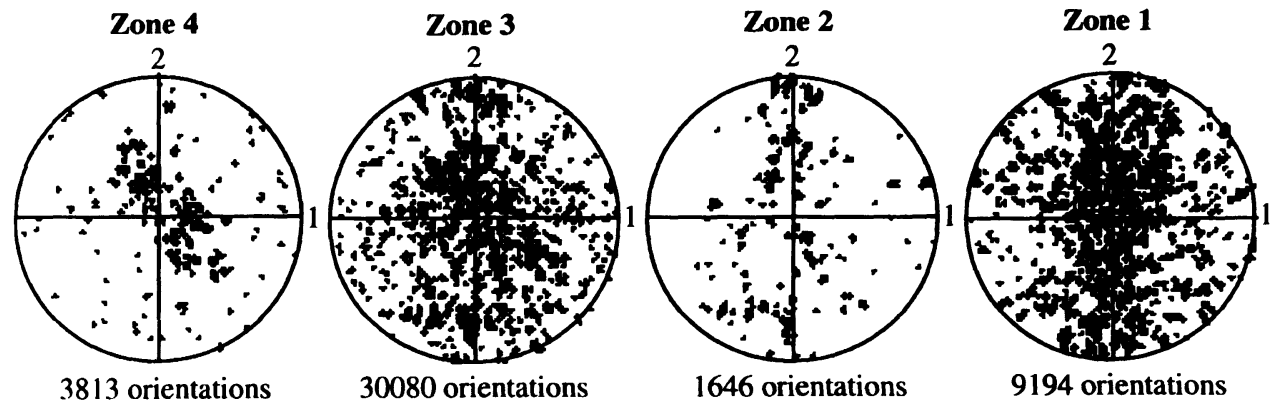

Figure 7 Discrete (0001) pole figures for the four zones. The weld line (WL) direction is vertical. 
Table 2 Summary of ODF calculations.

\begin{tabular}{cccc}
\hline zone & $\begin{array}{c}\text { texture } \\
\text { index }\end{array}$ & $\begin{array}{c}\text { maximum } \\
\text { intensity } \\
\text { (times random) }\end{array}$ & $\left(\varphi_{1}, \Phi, \varphi_{2}\right)$ \\
\hline 1 & 1.13 & 3.0 & $130^{\circ}, 35^{\circ}, 65^{\circ}$ \\
2 & 1.51 & 7.8 & $225^{\circ}, 40^{\circ}, 15^{\circ}$ \\
3 & 1.89 & 14.8 & $135^{\circ}, 20^{\circ}, 65^{\circ}$ \\
4 & 2.24 & 16.2 & $110^{\circ}, 55^{\circ}, 50^{\circ}$ \\
\hline
\end{tabular}

apparent growth direction in each zone). Let these directions be denoted $a_{2}, a_{3}$, and $a_{4}$ for zones 2, 3 and 4 respectively (these directions are assumed to lie in the metallographic section plane). The calculated inverse pole figures for these directions are shown in Figure 8 along with an indication of the apparent growth direction in each zone as measured from Figure 3 . These inverse pole figures are of moderate intensity (maximum equals 3.8 times random). The preferred crystallographic growth direction appears to be $<10 \overline{10} 0$.

The alignment of $\langle 10 \overline{1} 0>$ axes in the sample can be examined by looking at the $<10 \overline{1} 0>$ pole figures as shown in Figure 9. Each successive inverse pole figure (proceeding from zone 2 to zone 4 ) shows a stronger alignment of the $<10 \overline{1} 0>$ crystal directions. Therefore, it appears that grains which are initially favorably oriented for growth, i.e. those with their $<10 \overline{1} 0>$ axes aligned with $a_{3}$, simply outpace those which are not. There also appears to be slight tendency for the $<1010\rangle$ crystal directions to follow the changing direction of the thermal gradient.

Dividing zone 3 into three equal sub-zones (3a, $3 b$ and $3 c$ moving from right to left in zone 3 ) and plotting the $<10 \overline{10}>$ pole figures for these sub-zones shows this evolution to be gradual as can be seen in Figure 10.

The statistical distribution of misorientation was calculated for zone 1 and for zones 2,3 and 4 collectively. The space of the misorientation angle was partitioned into $5^{\circ}$ bins. The misorientation distribution for a given set of misorientations was determined by incrementing the appropriate bin according to the misorientation associated with each

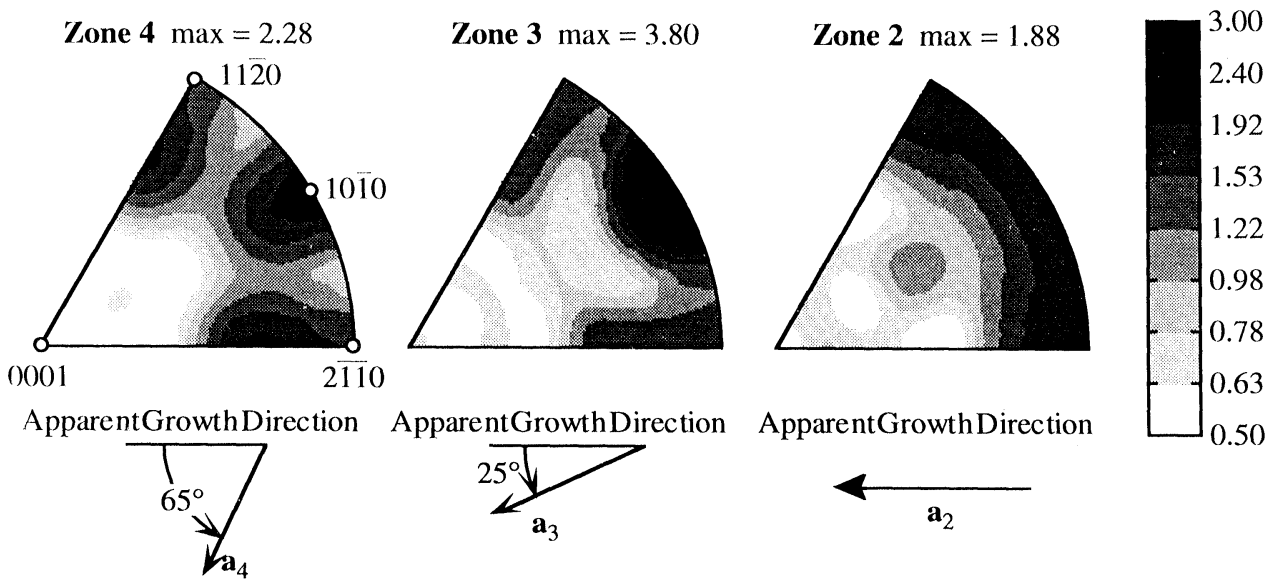

Figure 8 Apparent growth direction inverse pole figures for zones 2, 3 and 4. 

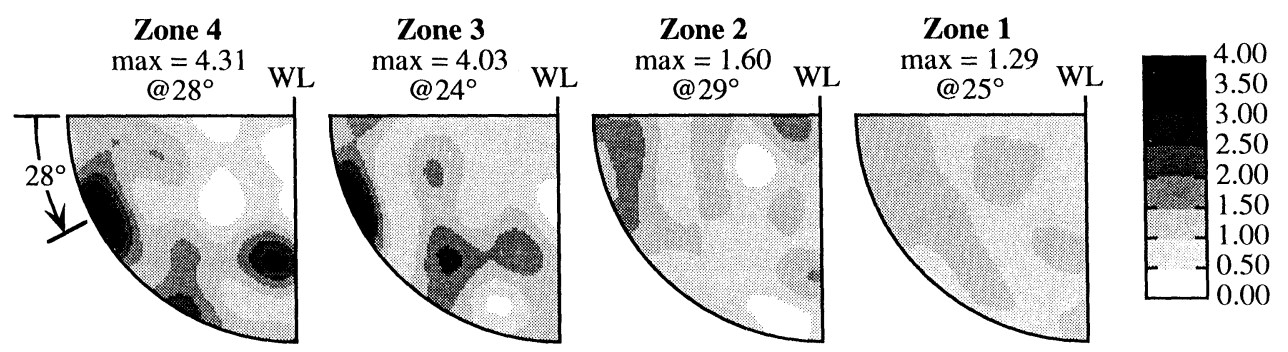

Figure $9<10 \overline{1} 0>$ pole figures for zones $1,2,3$ and 4 of the weld.

boundary. The bins were then normalized to the total number of boundaries in the set. The results of these calculations are shown in Figure 11. The complete three dimensional misorientation distribution functions (MDF) (not just the scalar misorientation angle as shown in Figure 11, but including the axis of rotation as well) for zone 1 and for the collective zones 2, 3 and 4 were also calculated using harmonic analysis. Both MDFs showed random rotations about the [0001] crystal axis (i.e. neighboring grains tend to have their [0001] axes nearly parallel and differ simply by rotations about [0001]).

An inverse pole figure was calculated for a direction normal to the weld fracture plane from the orientation measurements lying in zone 4 and is shown in Figure 12. The most intense peaks in Figure 12 occur at the approximate crystallographic directions of $\langle 4 \overline{2} \overline{2} 1>$.

However, the region of high intensity also spreads onto the $\langle 11 \overline{2} 0\rangle$ direction, which is normal to the $\{11 \overline{2} 0\}$ plane, a secondary fracture plane for beryllium when $\{0001\}$ planes are unfavorable (Darwin and Buddery, 1960). In fact, $12 \%$ of the grains have their $\langle 11 \overline{2} 0\rangle$ directions normal to the weld line while only $0.05 \%$ of the $<0001\rangle$ directions are within $10^{\circ}$ of the weld line normal. This is logical, since the preferred growth direction, $\langle 10 \overline{1} 0\rangle$, is coincident with the $\{0001\}$ planes, and the $\{0001\}$ planes are therefore at least $65^{\circ}$ away from the observed fracture plane and largely unavailable. Furthermore, the local fracture plane may readily deviate $10^{\circ}$ or more from the observed macroscopic plane on the fracture surface which can partially account for the spread in apparent fracture plane.
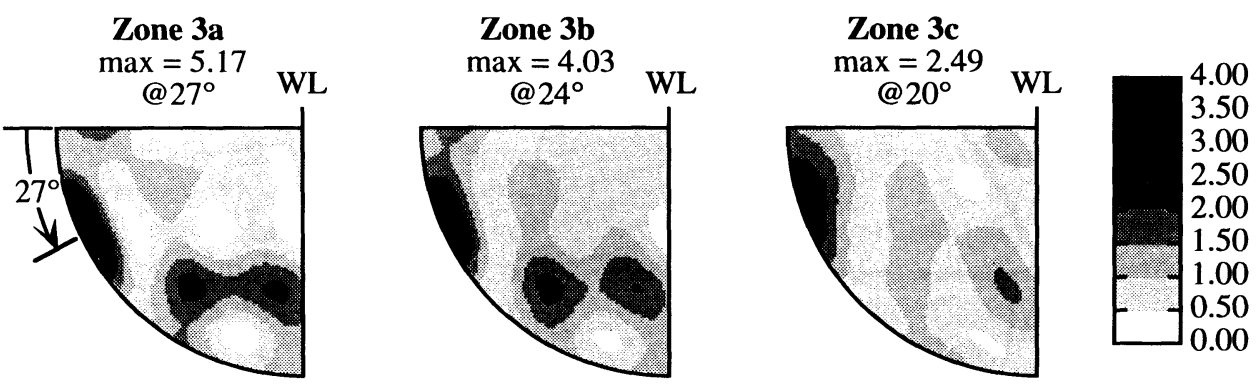

Figure $10<10 \overline{10}>$ pole figures for zones $3 \mathrm{a}, 3 \mathrm{~b}$ and $3 \mathrm{c}$. 


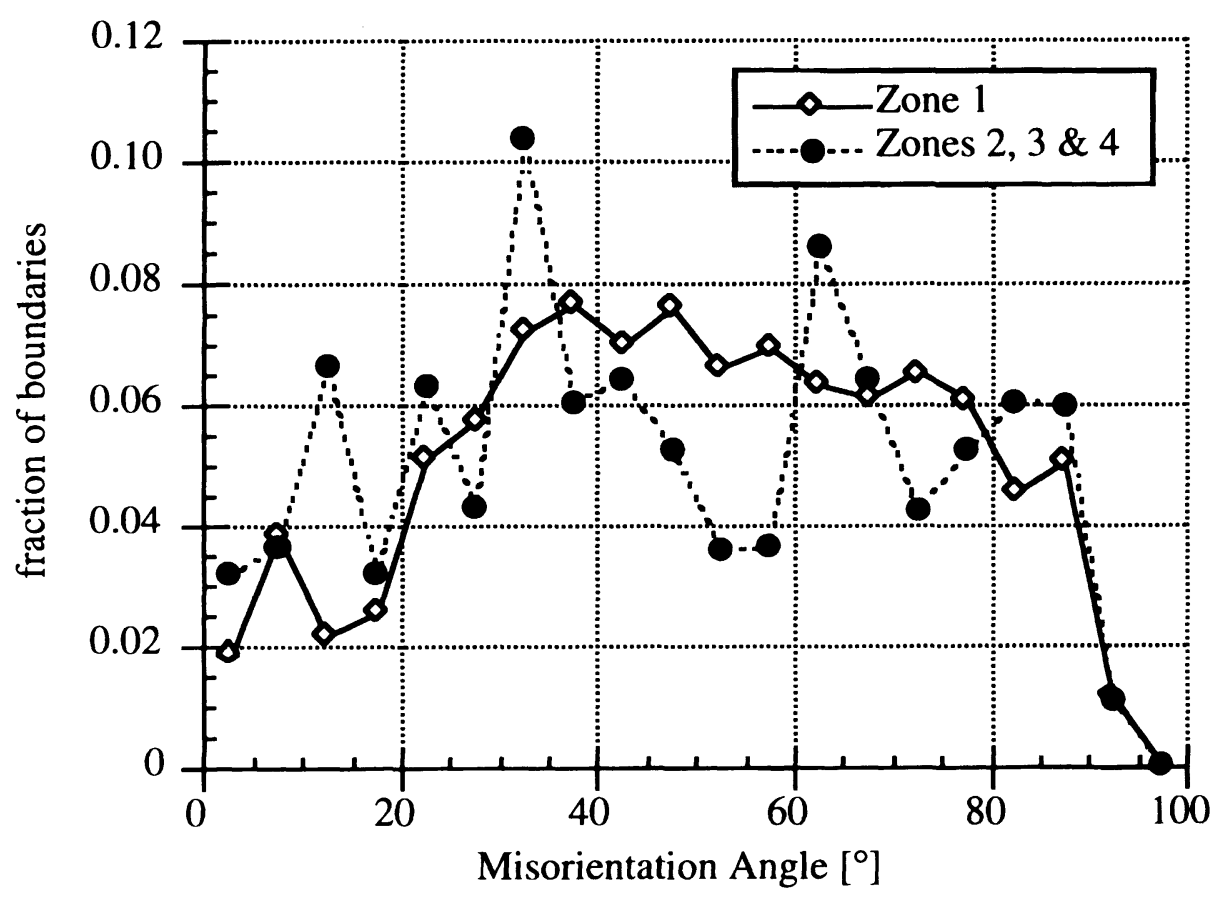

Figure 11 Misorientation angle distribution.
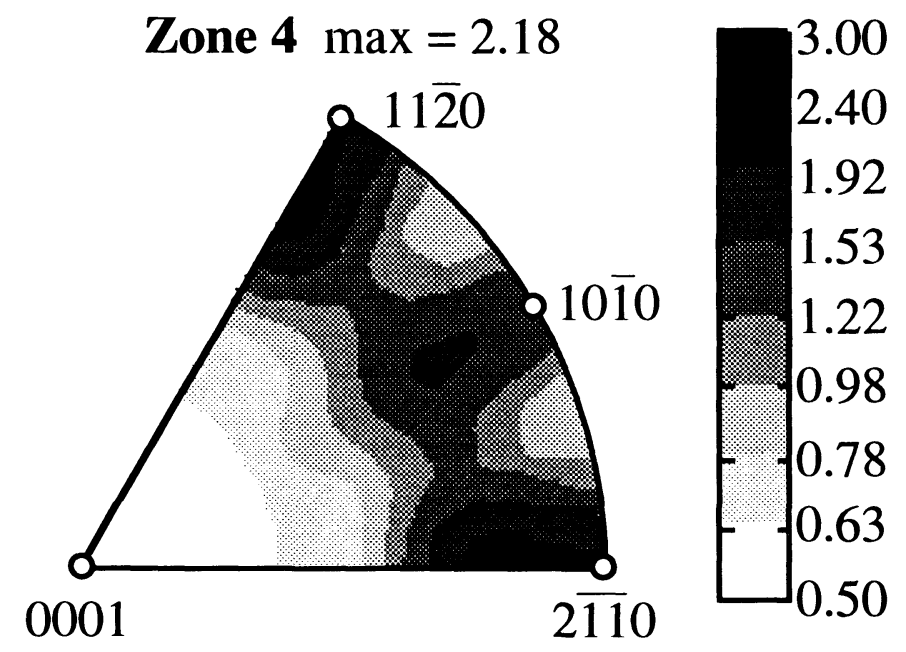

Figure 12 Zone 4 inverse pole figure for weld normal direction. 


\section{DISCUSSION}

\section{Individual Grain Misorientations}

One purpose of this study was to determine if grain bending occurs during the growth of high aspect grains due to the changing thermal gradient and impingement of the surrounding grains. This would be most evident by a gradual change in the crystallographic orientation of the long grains and should be measurable. The orientation measurements of sample grains A and B (Figures 3-6) show quite clearly that no significant bending occurs. Thus, it is evident that both local and long-range misorientations do not develop during growth and that significant plastic deformation has not taken place. However, since the apparent growth direction, i.e. the direction of the long dimension of the grains, has changed, this can only mean that the crystallographic growth direction shifts during growth.

\section{Grain Growth Selection}

The similarity between the textures of zones 1 and 2 indicates that the weld grains (zone 2) epitaxially nucleate from the base metal (zone 1) grains. From this set of grains, those grains with $<10 \overline{1} 0>$ crystallographic directions nearly parallel to a particular sample direction (denoted $a_{3}$ in the results section) outpace other grains as can be seen in zone 3 . The criticality of the growth direction $a_{3}$ likely arises from the magnitude and direction of the temperature gradient. There is evidence for a second critical point (at the boundary between zones 3 and 4) at which a particular subset of the grains which have managed to grow despite not having $<10 \overline{1} 0>$ crystal directions aligned with $a_{3}$ begin to outpace other grains in the set. As with the initial set of dominant growth grains, these grains tend to have their $\langle 10 \overline{1} 0>$ crystal directions aligned with a particular sample direction (denoted $a_{4}$ ) and form a second set of dominantly growing grains. However, this second set of grains is considerably smaller than the initial set, likely due to the confinement of the melt during the final stages of solidification. This secondary critical point does not appear to be as sharp in the transverse section micrograph as in the short-transverse section. The component of the temperature gradient normal to the plane of the coupon has been ignored since measurements were only collected from the short-transverse plane of the coupon. A more complete analysis would consider the transverse plane along with possibly other planes.

Misorientation calculations suggest that the selection of whether a given grain continues to grow is not coordinated with the local orientational relationships the given grain has with its nearest neighbors. The distribution of misorientation in the base metal (zone 1) is similar to that observed in the other zones, indicating that grains with certain types of neighborhoods are not more likely to grow over grains with other types of neighborhoods. Therefore, the selection of whether a given grain grows or not is more dependent on its orientation relative to the temperature gradient than its orientation relationships with its neighbors. The misorientation texture statistics are insufficient to elucidate possible second order neighborhood coordination effects.

\section{Fracture Plane}

One of the principle objectives of this work was to determine if the growth characteristics of the weld grains promote orientations which are favorable for centerline fracture, as suggested by Hauser et al. (1967). This scenario would require that planes of low fracture 
strength, $\{0001\}$ and $\{11 \overline{2} 0\}$, be approximately normal to the weld centerline. Figure 12 shows that this is the case to a limited extent. The tendency for growth to occur within the basal plane in direction $a_{3}$ (which is $65^{\circ}$ from the weld center line) essentially prevents $\{0001\}$ cleavage, yet allows $\{11 \overline{2} 0\}$ cleavage to a limited extent. Thus, to increase the centerline toughness of the weld would require minimization of these orientations. However, given the multiplicity of $\{11 \overline{2} 0\}$ planes and the rotational freedom of the grain orientations about the growth direction, this seems rather impractical.

\section{CONCLUSIONS}

The local grain orientations in a Be weldment have been characterized in detail. From analysis of the orientations the following conclusions have been drawn:

1) While the macroscopic growth direction changes during solidification, the crystallographic orientation does not. That is, substantial deformation of the weld grains does not occur.

2) The weld grains appear to be epitaxially nucleated from the base metal.

3) After nucleation, grains with $<1010>$ crystal directions aligned with the macroscopic growth direction tend to grow more quickly than others. No evidence was found for coordinated growth between neighboring grains.

4) The macroscopic fracture plane correlates best with $\{11 \overline{2} 0\}$ crystallographic planes, which are known to below cleavage strength planes in Be.

In order to better characterize the relationship of crystallographic orientation with microstructural features, similar microtextural investigations should be made on additional planes. Even though a considerable number of orientations were measured $(\sim 45,000)$ the number of actual grains sampled was only 589 . The number of grains sampled could be increased by simply increasing the coarseness of the measurement grid.

\section{Acknowledgments}

The authors acknowledge helpful discussions with A. D. Rollett and D. J. Thoma of Los Alamos National Laboratory and with M. J. Kaufman of the University of Florida. The welding was performed at Los Alamos National Laboratory by F. M. Smith. K. Kunze and B. L. Adams are gratefully acknowledged for allowing the authors access to the automatic single orientation measurement facility at Brigham Young University.

\section{References}

Adams, B. L., Wright, S. I. and Kunze, K. (1993). Orientation Imaging: The Emergence of a New Microscopy. Metall. Trans., 24A, 819-831.

Aldinger, F. (1979). Flow and Fracture of Single Crystals. In Beryllium Science and Technology, Vol. 1, edited by D. Webster and G. J. London, pp. 104. New York: Plenum Press.

Bunge, H.-J. (1982). Texture Analysis in Materials Science, Mathematical Methods, London: Butterworths.

Darwin, G. E. and Buddery, J. H. (1960). Beryllium, New York: Academic Press.

Grant, L. A. (1979). Joining II: Brazing and Soldering. In Beryllium Science and Technology, Vol. 2, edited by D. R. Floyd and J. N. Lowe, pp. 231-295. New York: Plenum Press.

Hauser, D., Mishler, H. W., Monroe, R. E. and Martin, D. C. (1976). Electron Beam Welding of Beryllium. Welding Journal, 525s-540s. 
Olson, D. L. and Liby, A. L. (1979). Joining III: Diffusion Bonding. In Beryllium Science and Technology, Vol. 2, edited by D. R. Floyd and J. N. Lowe, pp. 231-295. New York: Plenum Press. Switz, R. J. (1979). Joining I: Mechanical/Adhesive. In Beryllium Science and Technology, Vol 2, edited by D. R. Floyd and J. N. Lowe, pp. 231-247. New York: Plenum Press.

Wagner, F., Wenk, H. R., Esling, C. and Bunge, H. J. (1981). Importance of Odd Coefficients in Texture Calculations for Trigonal-Triclinic Symmetries. Phys. Stat. Sol. (b), 67, 269-285.

Wright, S. I. (1993). A Review of Automated Orientation Imaging Microscopy (OIM). J. Computer Assisted Microscopy, 5, 207-221. 